\title{
INFLUENCE OF THE FILLING GAS MIXTURE ON THE INTERRUPTION CAPABILITY OF MEDIUM VOLTAGE LOAD BREAK SWITCHES
}

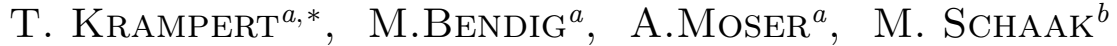 \\ ${ }^{a}$ Institute for High Voltage Equipment and Grids, Digitalization and Power Economics, RWTH Aachen \\ University, Schinkelstr. 6, 52056 Aachen, Germany \\ ${ }^{b}$ Siemens AG, Smart Infrastructure Distribution Systems, Carl-Benz-Str. 22, 60386 Frankfurt am Main, \\ Germany \\ * t.krampert@iaew.rwth-aachen.de
}

\begin{abstract}
In today's medium voltage switchgear $\mathrm{SF}_{6}$ is commonly used as insulating and arc quenching medium. Because of its high potential impact on the environment a substitution with an environmentally friendly alternative is pursued. In this paper the influence of the mixing ratio of carbon dioxide nitrogen mixtures as filling gas on the interruption capability in medium voltage load break switches is investigated. The interruption capability is regarded by means of the thermal interruption performance as well as the dielectric recovery of a model load break switch. The model load break switch allows an axial arc blowing with variable pressure and uses an exchangeable nozzle system.
\end{abstract}

Keywords: load break switch, alternative gas, thermal interruption capability, dielectric recovery.

\section{Introduction}

In the medium voltage (MV) secondary distribution a large number of load break switches (LBS) is installed to interrupt load currents up to $1250 \mathrm{~A}$ and as disconnector switches. Most of them are installed in ring main units (RMU) connecting the low voltage grid to the MV grid. Because of the limited space in urban areas, they are often designed as metal clad gas insulated switchgear (GIS). Sulphur hexafluoride $\left(\mathrm{SF}_{6}\right)$ with its superior insulating and arc quenching performance is the state of art filling gas for GIS. However, with a Global Warming Potential (GWP) of 23500 it is the most potent greenhouse gas known and therefore listed in the Kyoto protocol as one of the gases to be reduced $[1,2]$. Possible substitutes for $\mathrm{SF}_{6}$ should have a low GWP and no Ozone Depletion Potential (ODP). They must have a sufficient dielectric strength to insulate occurring voltage stress and a good arc quenching capability to guarantee current interruption. To ensure a safe operation over the complete service life the gas should be stable under the influence of an arc and not produce conducting byproducts [1]. For the use in MV switchgear different gas mixtures of atmospheric gases like carbon dioxide $\left(\mathrm{CO}_{2}\right)$ and nitrogen $\left(\mathrm{N}_{2}\right)$ with and without fluorinated gases are discussed [1, 3-5].

The arc quenching capability of a gas can be evaluated by means of the arcing time constant. The lower arcing time constant of $\mathrm{CO}_{2}$ indicates a better thermal interruption capability compared to $\mathrm{N}_{2}$ (see Table 1) [3]. By using $\mathrm{CO}_{2}-\mathrm{N}_{2}$ mixtures the impulse withstand voltage can be increased compared to pure $\mathrm{N}_{2}$ and pure $\mathrm{CO}_{2}[4,6]$. Both gases have no or a low GWP and no ODP (see Table 1). Investigations showed that $\mathrm{CO}_{2}$ can produce conducting byproducts

\begin{tabular}{lccc}
\hline \hline Property & $\mathrm{SF}_{6}$ & $\mathrm{CO}_{2}$ & $\mathrm{~N}_{2}$. \\
\hline GWP & 23500 & 1 & 0 \\
Dielectric strength $[\%]$ & 100 & 37 & 40 \\
Arcing time $[\mu \mathrm{s}]$ & 0.8 & $1.5-15$ & $210-220$ \\
\hline \hline
\end{tabular}

Table 1. Properties of $\mathrm{SF}_{6}, \mathrm{CO}_{2}$ and $\mathrm{N}_{2}[1,9]$

when interrupting large currents in the range of tens of $\mathrm{kA}[3]$.

$\mathrm{N}_{2}$ has a lower permeation rate than $\mathrm{CO}_{2}$ [7], which makes the enclosure design easier. By using a $\mathrm{CO}_{2}-$ $\mathrm{N}_{2}$ mixture, the advantages of both gases can be combined. Therefore, in this paper the influence of the mixing ratio of $\mathrm{CO}_{2}-\mathrm{N}_{2}$ mixtures on the interruption capability of a MV LBS is investigated. Some of the experimental results have been shown in [8] and are used for comparison in this work.

\section{Methodology}

The interruption process in LBS can be divided into a thermal and a dielectric phase [10]. To evaluate the influence of the filing gas mixture on both phases separately two different test methods are used, which are desribed shortly in the following. A detailed explanation can be found in [5] and [8].

\subsection{Thermal Interruption Capability}

In this paper, the thermal interruption capability is quantified by the critical current steepness $\mathrm{d} i / \mathrm{d} t_{\text {crit }}$. For the determination a synthetic test circuit is used. The test circuit provides one half oscillation of a sinusoidal current with a value of $I_{\mathrm{RMS}}=630 \mathrm{~A}$ and a frequency of $f_{1}=50 \mathrm{~Hz}$. Shortly before the current reaches its natural $\mathrm{CZ}$ an injection current in the range 


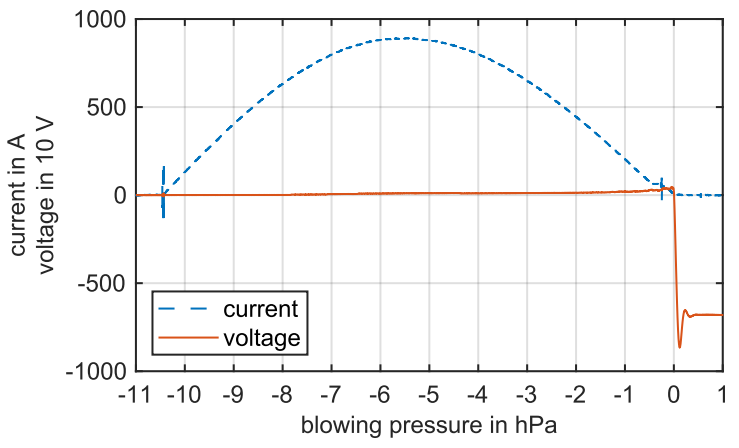

Figure 1. Voltage and current measurement of a succesfull interruption

of a few ten amps and a frequency of $f_{2} \approx 1000 \mathrm{~Hz}$ is superimposed on the test current. Using this superposition method, the current steepness in the model switch at $\mathrm{CZ}$ can be varied independently of the test current amplitude. After successful current interruption, a recovery voltage rises across the open contacts (see figure 1). To ensure a conservative test the rate of rise of the recovery voltage (RRRV) is chosen to create a RRRV corresponding to 1.2 times the RRRV of a standardized test for $I_{\mathrm{N}}=630 \mathrm{~A}$ at $U_{\mathrm{N}}=24 \mathrm{kV}$ and $f=50 \mathrm{~Hz}$. This results in an RRRV of approximately $\mathrm{d} u / \mathrm{d} t \approx 75 \mathrm{~V} /$ us at a current steepness of $\mathrm{d} i / \mathrm{d} t=0.28 \mathrm{~A} / \mathrm{\mu s}$ at CZ. At the beginning of a test series a low $\mathrm{d} i / \mathrm{d} t$ is chosen and five successful interruption tests are performed. Afterwards the $\mathrm{d} i / \mathrm{d} t$ is increased and another five interruptions are performed. This procedure is repeated until all five interruptions for one level of $\mathrm{d} i / \mathrm{d} t$ fail. The critical current steepness is determined as the mean value of the current steepness of the lowest failed test and the current steepness of the next lower successful test.

\subsection{Dielectric Recovery}

The dielectric recovery of the model switch is determined with the test method and circuit described in [8]. Firstly, the model switch is stressed with a half oscillation of a current with a value of $I_{\mathrm{RMS}}=630 \mathrm{~A}$ like in figure 1. Following the $\mathrm{CZ}$ a steep impulse voltage is applied after a short delay time. The impulse voltage has a maximum peak of $80 \mathrm{kV}$. The rise time ( $10 \%$ to $90 \%$ of the peak voltage) is $1 \mu \mathrm{s}$. The delay time is varied in a range of $t_{\text {delay }}=10 \mu \mathrm{s}$ to $300 \mu$ s after $\mathrm{CZ}$ to get a time resolved breakdown voltage after current zero. For each delay time a minimum of four breakdown tests is performed. In the resulting diagram each marker indicates the mean breakdown voltage of at least four measurements in a time frame of maximum $10 \mu \mathrm{s}$. The error bars indicate the maximum and minimum of the measured breakdown voltages in that time frame.

\subsection{Test Setup and Procedure}

The experiments presented in this paper are performed using a model switch with an axial blowing of the arc

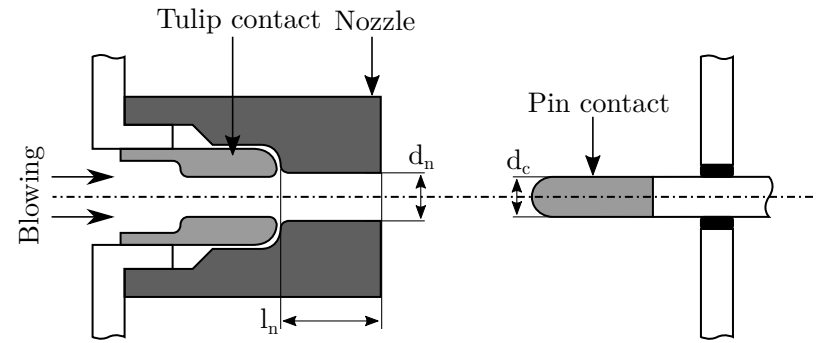

Figure 2. Section of the model load break switch [5]

through a polymer nozzle shown in figure 2 . It is build in a closed test vessel with a volume of $V=153 \mathrm{l}$. The mixing ratio is given by the volume fraction of $\mathrm{N}_{2}$. An absolute filling pressure of $p=1800 \mathrm{hPa}$ is used in all experiments. The model switch consists of a tulip-pin contact system made from tungsten-copper (80/20). The contact diameter is $d_{\mathrm{c}}=10 \mathrm{~mm}$. The used polymer nozzle is made from Polytetrafluorethylen (PTFE) and has a nozzle throat diameter of $d_{\mathrm{n}}=11 \mathrm{~mm}$ and a throat length of $l_{\mathrm{n}}=24 \mathrm{~mm}$. This configuration was identified as suitable in previous works $[5,8]$. The blowing through the tulip contact is realized by an external pressure vessel, which is filled with the same gas mixture as the test vessel. The opening of a magnetic valve initiates the blowing. The blowing pressure is measured upstream of the nozzle and gives the total pressure drop across tulip contact and nozzle. It is varied in a range from $p_{\mathrm{B}}=100 \mathrm{hPa}$ to $770 \mathrm{hPa}$. A pneumatic piston drive is used to operate the model switch with an average opening speed of $v_{\text {mean }}=5 \mathrm{~m} / \mathrm{s}$. Since in the used test circuit only current interruption in the first $\mathrm{CZ}$ can be tested and previous investigations showed that the interruption capability increases when the pin contact is outside of the nozzle at $\mathrm{CZ}$ [11] all experiments are conducted with an arcing time of $t_{\mathrm{Arc}} \geq 7 \mathrm{~ms}$.

\section{Results}

\subsection{Thermal Interruption Capability}

The critical current steepness for different $\mathrm{CO}_{2}-\mathrm{N}_{2}$ mixtures in dependence of the blowing pressure and the $\mathrm{N}_{2}$ content is shown in figure 3 . The lines connecting the data points are only drawn for better visualization. The used test circuit can create a current steepness of maximum $\mathrm{d} i / \mathrm{d} t_{\max }=1 \mathrm{~A} / \mu \mathrm{s}$. Therefore, the critical current steepness for the mixture with $20 \% \mathrm{~N}_{2}$ content can only be determined for blowing pressures $p_{\mathrm{B}} \leq 550 \mathrm{hPa}$ and for pure $\mathrm{CO}_{2}$ only for $p_{\mathrm{B}} \leq 330 \mathrm{hPa}$. For the measurement series with pure $\mathrm{N}_{2}$ the thermal interruption capability at a blowing pressure of $p_{\mathrm{B}}=100 \mathrm{hPa}$ is so low that no critical current steepness could be determined.

The critical current steepness increases with increasing blowing pressure and a decreasing $\mathrm{N}_{2}$ content. Mixtures with a $\mathrm{N}_{2}$ content of less than $80 \%$ show an almost linear increase with increasing blowing pressure in the investigated range. Mixtures with a $\mathrm{N}_{2}$ content 


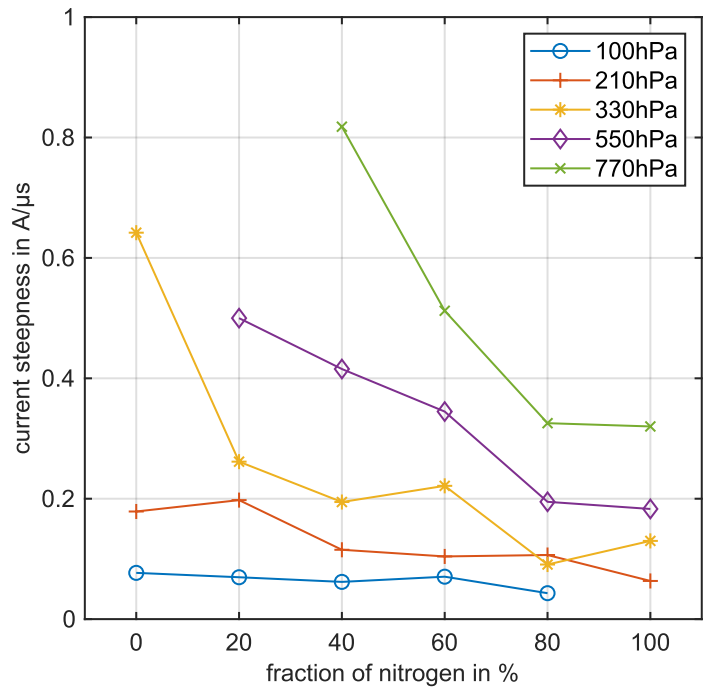

Figure 3. Critical current steepness depending on the fraction of $N_{2}$ for various blowing pressures

of $80 \%$ and more show an exponential increase of the critical current steepness with increasing blowing pressure.

For low blowing pressures, the $\mathrm{N}_{2}$ content has only a small effect on the critical current steepness. For a blowing pressure of $p_{\mathrm{B}}=770 \mathrm{hPa}$, a decreasing $\mathrm{N}_{2}$ content leads to an exponential increase of the critical current steepness.

\subsection{Dielectric Recovery}

Figure 4 shows the time resolved breakdown voltages for different $\mathrm{N}_{2}$ fractions at a blowing pressure of $p_{\mathrm{B}}=330 \mathrm{hPa}$. The differences between the different mixtures with $\mathrm{N}_{2}$ contents of $20 \%$ to $80 \%$ are small and in the area of the measured scatter. With the use of pure $\mathrm{CO}_{2}$, the model switch shows the fastest dielectric recovery. With pure $\mathrm{N}_{2}$ the breakdown voltage at $t_{\text {delay }} \approx 300 \mu$ s is less than $50 \%$ of the one measured with pure $\mathrm{CO}_{2}$.

The time resolved breakdown voltages for varying blowing pressures and a mixture of $40 \% \mathrm{~N}_{2}$ and $60 \%$ $\mathrm{CO}_{2}$ are shown in figure 5 . With increasing blowing pressure the dielectric recovery becomes faster. At $p_{\mathrm{B}}=330 \mathrm{hPa}$ the breakdown voltage increases almost linearly in the investigated time frame. For a blowing pressure of $p_{\mathrm{B}}=550 \mathrm{hPa}$ the dielectric recovery rises in the first $200 \mu$ s after $\mathrm{CZ}$ and then stays at a value around $U_{\mathrm{BD}} \approx 70 \mathrm{kV}$. For $p_{\mathrm{B}}=770 \mathrm{hPa}$ the breakdown voltage increases even faster in the first $150 \mu \mathrm{s}$ after CZ and increases further until the end of the investigated time frame.

\section{Discussion}

\subsection{Thermal Interruption Capability}

At low blowing pressures, the $\mathrm{N}_{2}$ content has only a small influence on the critical current steepness. It is assumed that through nozzle ablation a pressure

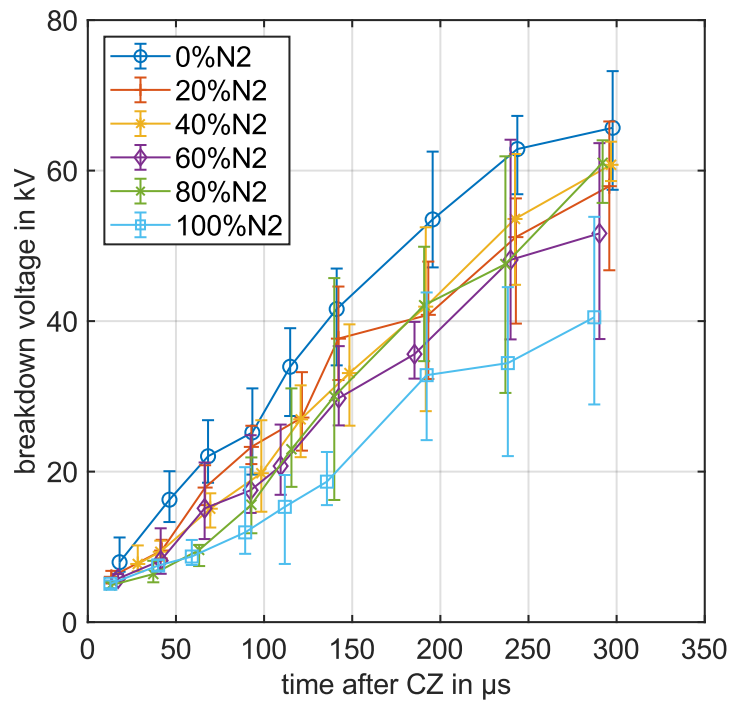

Figure 4. Dielectric Recovery of the model switch for various $\mathrm{N}_{2}-\mathrm{CO}_{2}$ mixtures at $p_{B}=330 \mathrm{hPa}$

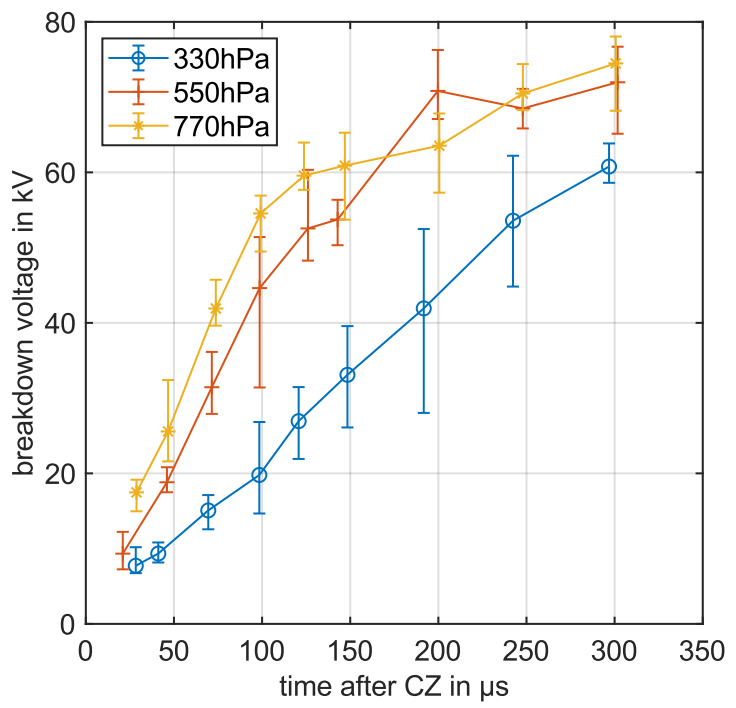

Figure 5. Dielectric Recovery of the model switch with mixture of $40 \% \mathrm{~N}_{2}$ and $60 \% \mathrm{CO}_{2}$ for various blowing pressures

rise inside the nozzle occurs, which leads to a clogging of the nozzle, resulting in an insufficient flow field. Additionally, this results in an unknown gas mixture in the nozzle where the $\mathrm{N}_{2}$ content has a minor effect on the thermal interruption capability compared to high blowing pressure. At higher blowing pressure the flow field at $\mathrm{CZ}$ is stable and the $\mathrm{N}_{2}$ content of the gas mixture has a greater influence on the thermal interruption capability. The higher interruption capability for lower $\mathrm{N}_{2}$ contents results from the smaller arcing time constant of $\mathrm{CO}_{2}$ (see Table 1). At comparable cooling, by axial blowing, the resistance of the switching gap rises faster for $\mathrm{CO}_{2}$. This results in a faster limitation of the post arc current, which leads to a decreased heating and therefore to a decreased need 
for cooling. The authors assume that the significantly higher thermal conductivity of $\mathrm{CO}_{2}$ compared with $\mathrm{N}_{2}$ in the temperature range from $2000 \mathrm{~K}$ to $5000 \mathrm{~K}$ leads to the faster cooling of the gas in the switching gap. Combined with the limitation of the post arc current and therefore decreased post arc heating this results in the exponential increase in thermal interruption capability with an increasing $\mathrm{CO}_{2}$ content.

\subsection{Dielectric Recovery}

The higher thermal conductivity of $\mathrm{CO}_{2}$ leads to a faster cooling of the gas in the switching gap and therefore to a faster dielectric recovery in the first $300 \mu$ s after CZ. With an increased blowing pressure the cooling can also be intensified and a faster dielectric recovery is achieved. The faster dielectric recovery of switchgear using $\mathrm{CO}_{2}$ in comparison to $\mathrm{N}_{2}$ is also reported in literature [12].

In $[4,6]$ an increase in breakdown voltage of $\mathrm{CO}_{2}$ $\mathrm{N}_{2}$ mixtures compared with pure $\mathrm{CO}_{2}$ and pure $\mathrm{N}_{2}$ is identified. While in $[4,6]$ tests without prior stress through a switching arc were performed, this work investigates the breakdown voltage of the gases after current interruption. Therefore the gas is still hot when the breakdown voltage is determined. Hence, these results are not directly comparable to the cold gas breakdowns. The dielectric recovery of all tested gases and gas mixtures for $t_{\text {delay }} \geq 200 \mu \mathrm{s}$ is sufficient to withstand the recovery voltage at $24 \mathrm{kV}$ load current interruption. For $t_{\text {delay }}<200 \mu$ s the dielectric recovery of mixtures with a low $\mathrm{CO}_{2}$ content might lead to breakdowns in the first peak of the recovery voltage. The investigations on the influence of the blowing pressure show that in this time frame the dielectric recovery can be significantly accelerated by an increase of the blowing pressure. Since in the test method for determining the thermal interruption capability the recovery voltage is replicated until the first maximum of the recovery voltage, all configurations, which have a sufficient thermal interruption capability, will also have a sufficient dielectric strength in the critical time frame.

\section{Conclusion}

The thermal interruption capability and dielectric recovery of a model LBS is investigated for $\mathrm{CO}_{2}-\mathrm{N}_{2}$ mixtures. By increasing the content of $\mathrm{CO}_{2}$ in a gas mixture with $\mathrm{N}_{2}$, the thermal interruption capability can be improved compared to pure $\mathrm{N}_{2}$. With a LBS using pure $\mathrm{N}_{2}$ the required interruption capability of $\mathrm{d} i / \mathrm{d} t=0.28 \mathrm{~A} / \mu \mathrm{s}$ for a the interruption of a $630 \mathrm{~A}$ load current in the $24 \mathrm{kV}$ voltage level is achieved with a blowing pressure of $770 \mathrm{hPa}$. When using pure $\mathrm{CO}_{2}$ only a blowing pressure of $330 \mathrm{hPa}$ is necessary. The dielectric recovery is also influenced positively by an addition of $\mathrm{CO}_{2}$. From the presented investigations it can be expected that when a configuration has a sufficient thermal interruption capability the dielectric recovery will also be sufficient. In field application, the blowing pressure is generated by the compression of the filling gas in a puffer volume. Therefore, the choice of the gas mixture allows for an optimization of the needed drive.

\section{References}

[1] M. Seeger, R. Smeets, J. Yan, et al. Recent development of alternative gases to $\mathrm{SF}_{6}$ for switching applications. Electra, 291, 2017.

[2] United Nations. Kyoto protocol to the united nations framework convention on climate change, 1998.

[3] T. Uchii, Y. Hoshina, H. Kawano, et al. Fundamental research on $\mathrm{SF}_{6}$-free gas insulated switchgear adopting $\mathrm{CO}_{2}$ gas and its mixtures. In International Symposium on Eco Topia Science, pages 516-520, 2007.

[4] M. Bendig, N. Götte, T. Krampert, et al. A method to determine the rate of the dielectric recovery in a medium voltage load break switch with a free burning switching arc. In 22nd International Conference on Gas Discharges and Their Applications, 2018.

[5] M. Bendig, T. Krampert, N. Götte, et al. Investigations on the effect of the nozzle material on the interruption capability of a medium voltage load break switch. Plasma Physics and Technology, 6(1):15-18, 2019. doi:10.14311/ppt.2019.1.15.

[6] P. G. Nikolic, A. Kurz, M. Hoffacker, and A. Schnettler. Investigations on the dielectric strength of carbon dioxide and carbon dioxide mixtures for the application in gas insulated switchgear. In IEEE International Power Modulator and High Voltage Conference, 2012.

[7] P. Sturm, M. Leuenberger, C. Sirignano, et al. Permeation of atmospheric gases through polymer o-rings used in flasks for air sampling. Journal of Geophysical Research: Atmospheres, 109(D4), 2004. doi:10.1029/2003JD004073.

[8] M. Bendig and M. Schaak. Design rules for environmentally friendly medium voltage load break switches. IEEE Transactions on Power Delivery, 2020. doi:10.1109/TPWRD . 2020.3024983.

[9] CIGRE. Dry air, $\mathrm{N}_{2}, \mathrm{CO}_{2}$ and $\mathrm{N}_{2}-\mathrm{SF}_{6}$ mixtures for gas-insulated systems: Technical brochure 730 .

[10] K. Niayesh and M. Runde. Power Switching Components: Theory, Applications and Future Trends. Power Systems. Springer International Publishing, 2017. ISBN 9783319514598. doi:10.1007/978-3-319-51460-4.

[11] E. Jonsson, N. S. Aanensen, and M. Runde. Current interruption in air for a medium-voltage load break switch. IEEE Transactions on Power Delivery, 29(2):870875, 2014. doi:10.1109/TPWRD. 2013. 2280300.

[12] N. Götte, M. Bendig, T. Krampert, et al. Experimental and simulative study on the influence of the electrical field distribution on the dielectric switching behavior of natural gases. In 22nd International Conference on Gas Discharges and Their Applications, 2018. 